BULL. AUSTRAL. MATH, SOC.

VOL. 36 (1987) 353-360

\title{
ON THE KNESER-HUKUHARA PROPERTY FOR \\ INTEGRAL EQUATIONS IN LOCALLY CONVEX SPACES
}

\author{
STANISLAW SZUFLA
}

This paper contains a Hukuhara - type theorem for nonlinear volterra integral equations in locally convex spaces.

1. Introduction

Let $T=\{(t, s): 0 \leq s \leq t \leq a\}$ and let $W$ be an open subset of a quasicomplete locally convex topological vector space $E$. In this paper we consider the integral equation

$$
x(t)=q(t)+\int_{0}^{t} f(t, s, x(s)) d s,
$$

where $f$ is a bounded continuous function from $T \times W$ into $E$ and $q$ is a continuous function from $[0, a]$ into $W$. We shall give sufficient conditions for the existence of a continuous solution of ( 1 ). These conditions are formulated in terms of measures of noncompactness (see [9]). In particular, they hold in the case when $f=f_{1}+f_{2}$, where $f_{1}$ is completely continuous and $f_{2}$ satisfies a Kamke condition (see [5]). Moreover, we shall show that the set of all continuous solutions of (1) is a continuum in the corresponding space of continuous functions. For the case when $E$ is a Banach space, similar problems were investigated in several papers (see [10], [8], [11], [13]). Our considerations are based on the Lema from Section 2 which gives a topological characterization of sets of fixed points of a certain class of nonlinear operators, Received 9 October 1986.

Copyright Clearance Centre, Inc. Serial-fee code: 0004-9729/87 $\$ \mathrm{~A} 2.00+0.00$. 
namely the so-called volterra operators introduced in [14]. Section 2 is a complement to the papers [12] and [3] (see also [14]), but our Lemma does not follow from these papers.

\section{The basic lemma}

Let $K$ be a bounded convex subset of a normed space and let $Y$ be a Hausdorff topological vector space. Denote by $C=C(K, Y)$ the space of continuous functions $K \rightarrow Y$ with the topology of uniform convergence.

LEMMA. Asswe that $E: C \rightarrow C$ is a continuous mapping such that

1. the set $F(C)$ is equiuniformly continuous;

2. there exist $t_{0} \in K$ and $x_{0} \in Y$ such that

$$
F(x)\left(t_{0}\right)=x_{0} \text { for azz } x \in C \text {; }
$$

3. for every $\varepsilon>0$ and $x, y \in C$

$$
x\left|K_{\varepsilon}=y\right| K_{\varepsilon} \Rightarrow F(x)\left|K_{\varepsilon}=F(y)\right| K_{\varepsilon},
$$

where $K_{\varepsilon}=\left\{t \in K:\left\|t-t_{0}\right\| \leq \varepsilon\right\}$;

4. every sequence $\left(x_{n}\right)$ in $C$ such that $\lim _{n \rightarrow \infty}\left(x_{n}-F\left(x_{n}\right)\right)=0$ has a limit point.

Then the set $S_{F}=\{x \in C: x=F(x)\}$ is nonempty and connected whenever it is compact.

Proof. By Lemma 1 of [12] there exists a sequence ( $F_{n}$ ' such that $I-F_{n}$ is a homeomorphism $C \rightarrow C$ and $\lim _{n \rightarrow \infty} F_{n}(x)=F(x)$ uniformly in $x \in C$. From this and condition 4 it is clear that $S_{F} \neq \varnothing$. Suppose that $S_{F}$ is compact and not connected. Thus there are nonempty compact sets $S_{0}, S_{1}$ such that $S_{F}=S_{0} \cup S_{1}$ and $S_{0} \cap S_{1}=0$. As $C$ is a completely regular space, this implies (see [7], Chapter 4I, II, Remark 3) that there exists a continuous function $w: C \rightarrow[0,1]$ such that $w(x)=0$ for $x \in S_{0}$ and $w(x)=1$ for $x \in S_{1}$. Fix $u_{0} \in S_{0}, u_{1} \in S_{1}$ and a positive integer $n$. Put

$$
e_{n}(r)=(1-r)\left(u_{0}-F_{n}\left(u_{0}\right)\right)+r\left(u_{1}-F_{n}\left(u_{1}\right)\right) \quad(0 \leq r \leq 1)
$$

and $u_{n r}=\left(I-E_{n}\right)^{-1}\left(e_{n}(r)\right)$. Since $e_{n}(r)$ depends continuously on $r$ 
and $I-F_{n}$ is a homeomorphism, the mapping $r+w\left(u_{n r}\right)$ is continuous on $[0,1]$. Moreover, $u_{n o}=u_{0}$ and $u_{n 1}=u_{1}$, so that $w\left(u_{n o}\right)=0$ and $w\left(u_{n 1}\right)=1$. From this we deduce that there exists $r_{n} \in[0,1]$ such that

$$
w\left(u_{n r_{n}}\right)=1 / 2
$$

For convenience put $v_{n}=u_{n r}$. As $\lim _{n \rightarrow \infty} e_{n}(r)=0$ uniformly in $r$ ! we get

$$
\lim _{n \rightarrow \infty}\left(v_{n}-F\left(v_{n}\right)\right)=\lim _{n \rightarrow \infty}\left(F_{n}\left(v_{n}\right)-F\left(v_{n}\right)+e_{n}\left(r_{n}\right)\right)=0 \text {. }
$$

Consequently, by condition 4 , the sequence $\left(v_{n}\right)$ has a limit point $v$. In view of (3) it is clear that $v \in S_{F}$, so that $w(v)=0$ or $w(v)=1$. On the other hand, (2) implies that $w(v)=1 / 2$, which yields a contradiction.

3. Volterra integral equations in locally convex spaces

Now we return to the equation (1). Let $P$ be a family of continuous seminorms generating the topology of $E$. For any $p \in P$ and for any bounded subset $X$ of $E$ denote by $\beta_{p}(X)$ the infimum of all $\varepsilon>0$ for which there exists a finite subset $Z$ of $E$ such that $X \subset 2+B_{p}(\varepsilon)$, where $B_{p}(\varepsilon)=\{x \in E: p(x) \leq \varepsilon\}$. The family $\left(B_{p}(X)\right)_{p \in P}$ is called the Hausdorff measure of noncompactness of $X$ (for properties see [9]).

Let us recall that a function $h: T \times R_{+} \rightarrow R_{+}$is called a Kamke function if $h$ satisfies the Caratheodory conditions and, for any $0<d \leq a$, the function $u=0$ is the unique nonnegative continuous solution of the inequality $u(t) \leq \int_{0}^{t} h(t, \varepsilon, u(s)) d s$ on $[0, d]$.

THEOREM 1. Assume that for any $p \in P$ there exists a Kamke function $(t, s, u) \rightarrow h_{p}(t, u)$ such that $h_{p}$ is nondecreasing in $u$ and

$$
B_{p}(f(t,[0, t] \times X)) \leq h_{p}\left(t, B_{p}(X)\right)
$$

for each $t \in[0, a]$ and for each bounded subset $X$ of $E$. 
Then there exists an interval $J=[0, d]$ such that the set of all continuous solutions $x: J \rightarrow E$ of (1), considered as a subset of $C(J, E)$, is nonempty, compact and connected.

Proof. As $W$ is open and $q$ is continuous, we can choose a set $B$ of the form $B=\left\{x \in E: p_{i}(x) \leq b, i=1, \ldots, m\right\}$, where $p_{1}, \ldots, p_{m}$ $\epsilon P$, such that $q(t)+B \subset W$ for all $t \in[0, a]$. Let $M=\sup \left\{p_{i}(f(t, 8, x)): i=1, \ldots, m,(t, s) \in T, x \in W\right\}$, $d=\min (a, b / M)$ and $J=[0, d]$. Put

$$
r(x)=\left\{\begin{array}{lll}
x & \text { for } & x \in B \\
x / Q(x) & \text { for } & x \in E \backslash B,
\end{array}\right.
$$

where $Q$ is the Minkowski functional of $B$, and put

$$
g(t, s, x)=f(t, s, q(s)+r(x-q(s))) \text { for }(t, s) \in T \text { and } x \in E \text {. }
$$

From the known properties of Minkowski's functional it follows that $r$ is a continuous function from $E$ into $B$ and $r(X) \subset \underset{0 \leq \lambda \leq 1}{U} \lambda X$ for each subset $X$ of $E$. Hence

$$
B_{p}(\{q(s)+r(x-q(s)): s \in[0, a], x \in X\}) \leq B_{p}(X)
$$

for any $p \in P$ and for any bounded subset $X$ of $E$. This shows that $g$ is a bounded continuous function from $T \times E$ into $E$ and $g$ satisfies (4).

$$
\begin{aligned}
& \text { We introduce a mapping } F \text { defined by } \\
& F(x)(t)=q(t)+\int_{0}^{t} g(t, s, x(s)) d s \text { for } t \in J \text { and } x \in C,
\end{aligned}
$$

where $C=C(J, E)$. It can be easily verified that $F$ is a continuous mapping $C+C$, the set $F(C)$ is bounded and equicontinuous, and (1) is equivalent to the equation $x=F(x)$. Now we shall show that $F$ satisfies condition 4 . Let $\left(x_{n}\right)$ be a sequence such that

$$
\lim _{n \rightarrow \infty}\left(x_{n}-F\left(x_{n}\right)\right)=0 \text {. }
$$

Put $V=\left\{x_{n}: n=1,2, \ldots\right\}$ and $V(t)=\{u(t): u \in V\}$. As the set $E(C)$ is equicontinuous, it follows from (5) that $V$ is also equicontinuous. Therefore for any $p \in P$ the function $t+v(t)=\beta_{p}(V(t))$ is 
continuous on $J$. For a given $t \in J$ we divide the interval $[0, t]$ into $n$ parts $0=t_{0}<t_{1}<\ldots<t_{n}=t$ in such a way that $\Delta t_{i}=t_{i}-t_{i-1}=t / n$ for $i=1, \ldots, n$.

Let $V_{i}=\left\{u(s): u \in V, t_{i-1} \leq s \leq t_{i}\right\}$. Then

$$
F(v)(t) \subset q(t)+\sum_{i=1}^{n} \Delta t_{i} \overline{\operatorname{conv}} g\left(t,[0, t] \times v_{i}\right)
$$

Moreover, for any $i, 1 \leq i \leq n$, there exists $\varepsilon_{i} \in\left[t_{i-1}, t_{i}\right]$ such that

$$
\beta_{p}\left(V_{i}\right)=\sup \left\{\beta_{p}(V(s)): t_{i-1} \leq s \leq t_{i}\right\}=v\left(s_{i}\right)
$$

(see [1], Theorem 2.2). Hence, by (5), (4) and corresponding properties of $B_{p}$, we obtain

$$
\begin{aligned}
v(t) \leq \beta_{p}(F(V)(t)) \leq \sum_{i=1}^{n} \Delta t_{i} \beta_{p}\left(g\left(t,[0, t] \times v_{i}\right)\right) & \leq \sum_{i=1}^{n} \Delta t_{i} h_{p}\left(t, \beta_{p}\left(V_{i}\right)\right) \\
& =\sum_{i=1}^{n} \Delta t_{i} h_{p}\left(t, v\left(s_{i}\right)\right) .
\end{aligned}
$$

But if $n \rightarrow \infty$, then $\sum_{i=1}^{n} \Delta t_{i} h_{p}\left(t, v\left(s_{i}\right)\right)+\int_{0}^{t} h_{p}(t, v(s)) d s$. Thus

$$
v(t) \leq \int_{0}^{t} h_{p}(t, v(s)) d s \text { for } t \in J \text {. }
$$

As $h_{p}$ is a Kamke function, this implies that

$$
{ }_{p}(V(t))=0 \text { for all } t \in J \text {. }
$$

Since this equality holds for every $p \in P$, it follows that for any $t \in J$ the set $V(t)$ is relatively compact in $E$. By Ascoli's theorem [6; Theorem 7.17] from this we deduce that $V$ is relatively compact in $C$. Hence the sequence $\left(x_{n}\right)$ has a limit point. On the other hand, as $S_{F}=F\left(S_{F}\right)$, by repeating the above argument we infer that $S_{F}$ is compact. Applying now the Lemma, we conclude that $S_{F}$ is nonempty and connected.

4. Kneser's theorem for weak solutions of the Cauchy problem In this section we shall present another application of the Lemma. Let $E$ be a (sequentially) weakly complete Banach space, $B=\{x \in E$ : $\left.\left\|x-x_{0}\right\| \leq b\right\}$, and let $f:[0, a] \times B \rightarrow E$ be a bounded weakly-weakly continuous function. Let $M=\sup \{\|f(t, x)\|: 0 \leq t \leq a, x \in B\}$, 
$d=\min (a, b / M)$ and $J=[0, d]$. Denote by $E_{w}$ the space $E$ provided with the weak topology.

THEOREM 2. Assume that

$$
B(f(J \times X)) \leq h(B(X)) \text { for each subset } X \text { of } B \text {, }
$$

where $h$ is a nondecreasing Komke function and $\beta$ is the measure of weak noncompactness (see [2]).

Then the set of all weak solutions of the Cauchy problem

$$
x^{\prime}=f(t, x), x(0)=x_{0},
$$

defined on $J$, is nonempty, compact and connected in $C(J, E)$.

Proof. Put

$$
r(x)=\left\{\begin{array}{l}
x \quad \text { if } x \in B \\
x_{0}+b\left(x-x_{0}\right) /\left\|x-x_{0}\right\| \text { if } x \in E \backslash B
\end{array}\right.
$$

and

$$
F(x)(t)=x_{0}+\int_{0}^{t} f(s, r(x(s))) d s \quad(t \in J, x \in C),
$$

where $C=C\left(J, E_{w}\right)$.

It is known that $F$ is a continuous mapping $C \rightarrow C$, the set $F(C)$ is bounded and equiuniformly (strongly) continuous, and the function $x \in C$ is a weak solution of (6) if and only if $x=F(x)$. Arguing similarly as in the proof of Theorem 1 , we can show that $F$ satisfies condition 4 and the set $S_{F}$ is nonempty and compact. By the Lemma it follows from this that $S_{F}$ is connected.

Remark. The assumptions of Theorem 1 or 2 guarantee that the corresponding operator $F$ satisfies condition 4 , but it is an open question whether it satisfies the stronger condition:

for every net $\left(x_{\alpha}\right)$

$\lim \left(x_{\alpha}-F\left(x_{\alpha}\right)\right)=0 \Longrightarrow\left(x_{\alpha}\right)$ has a limit point.

Therefore our theorems do not follow from the results of [3] and [12]. 


\section{References}

[1] A. Ambrosetti, "Un teorema di esistenza per le equazioni differenziali negli spazi di Banach", Rend. Sem. Mat. Univ. Padova, 39 (1967), 349 - 369.

[2] E. Cramer, V. Lakshmikantham and A. Mitchell, "On the existence of weak solutions of differential equations in nonreflexive Banach spaces", Nonlinear Anal. 2 (1978), 169-177.

[3] J. Dubois and P. Morales, "On the Hukuhara - Kneser property for some Cauchy problems in locally convex topological vector spaces", Lecture Notes in Math. 964 (1982), 162-170.

[4] M. Hukuhara, "Sur 1'application qui fait correspondre a un point un continu bicompact", Proc. Japan Acad. 31 (1955), 5-7.

[5] M. Hukuhara, "Théorems fondamentaux de la théorie des équations différentielles ordinaires dans l'espace vectoriel topologique", J. Fac. Sci. Univ. Tokyo, Sec.I., 8 (1959), 111-138.

[6] J.I. Kelley, General topology, (Toronto - New York - London 1957).

[7] K. Kuratowski, Topology, Vol.II, (New York - London - Warszawa 1968).

[8] V. Lakshmikantham, "Existence and comparison results for Volterra integral equations in a Banach space", Lecture Notes in Math. 737 (1979), 120-126.

[9] B.N. Sadovskii, "Limit-compact and condensing operators", Russian Math. Surveys, 27 (1972), 85-155.

[10] S. Szufla, "On the existence of solutions of Volterra integral equations in Banach spaces", Bulz. Acad. Polon. Sci. Ser. Sci. Math. 22 (1974), 1209-1213.

[11] S. Szufla, "On Volterra integral equations in Banach spaces", Funkcial. Ekvac. 20 (1977), 247-258.

[12] S. Szufla, "Sets of fixed points of nonlinear mappings in function spaces", Funkcial. Ekvac. 22 (1979), 121-126.

[13] R.L. Vaughn, "Criteria for the existence and comparison of solutions to nonlinear Volterra integral equations in Banach spaces", Nonlinear Equations in Abstract Spaces, ed. V. Lakshmikantham (New York 1978), 463-468. 
360

Stanis Yaw Szufla

[14] G. Vidossich, "A fixed point theorem for function spaces", J. Math. Anal. Appl. 36 (1971), 581-587.

A. Mickiewicz University

Poznán

Poland 